MNHMT2009-18291

\title{
DEVELOPMENT OF AN EFFICIENT PARTICLE APPROACH FOR MICRO-SCALE GAS FLOW SIMULATIONS
}

\author{
Quanhua SUN ${ }^{1}$, Feng $\mathrm{LI}^{1}$, Jing FAN ${ }^{1}$, Chunpei CAI ${ }^{2}$ \\ ${ }^{1}$ LHD, Institute of Mechanics, Chinese Academy of Sciences, Beijing 100190, China \\ ${ }^{2}$ Mechanical \& Aerospace Engineering, New Mexico State University, Las Cruces, NM 88003, USA
}

\begin{abstract}
The micro-scale gas flows are usually low-speed flows and exhibit rarefied gas effects. It is challenging to simulate these flows because traditional CFD method is unable to capture the rarefied gas effects and the direct simulation Monte Carlo (DSMC) method is very inefficient for low-speed flows. In this study we combine two techniques to improve the efficiency of the DSMC method. The information preservation technique is used to reduce the statistical noise and the cell-size relaxed technique is employed to increase the effective cell size. The new cell-size relaxed IP method is found capable of simulating micro-scale gas flows as shown by the 2D lid-driven cavity flows.
\end{abstract}

Keywords: direct simulation Monte Carlo method, information preservation method, cavity flow, micro-scale gas flow

\section{INTRODUCTION}

Gas flows at micro scale have recently gained intensive attention due to the rapidly developed microelectromechanical devices [1]. Study of these flows is important for both fundamental scientific issues and technological applications. For instance, early studies on micro-channel gas flow had indicated that the Navier-Stokes equations became invalid due to the rarefied gas effects existed in micro flows [2]. To enhance the design and performance of microelectromechanical systems, it is necessary to achieve deeper understanding of the related flow and thermal behaviors.

Gas flows can be categorized according to flow regimes using the Knudsen $(\mathrm{Kn})$ number: continuum flows when $\mathrm{K} n<0.01$, slip flows when $0.01<\mathrm{Kn} \leq 0.1$, transitional flows when $0.1<\mathrm{Kn} \leq 10$, and free molecular flows when $\mathrm{Kn}>10$. Micro flows are usually in the slip or transitional flow regimes.
Continuum flows can be described using the continuum equations such as the Navier-Stokes equations and the free molecular flows can be solved using the free-molecular theory. The slip and transitional flows, however, are very difficult to be simulated accurately and efficiently.

In the literature, many effects have been made to develop numerical approaches for modeling micro flows. One way is to extend the continuum equations by using slip boundary conditions or to include more terms in the continuum equations such as extended Navier-Stokes equations or Burnett equations. These continuum-based approaches are usually efficient, but their accuracy is not guaranteed for general flows. For instance, wall-slip boundary models are not truly predictive since the adjustable parameter is determined based on limited experimental measurements.

Another strategy is to improve the efficiency of kinetic approaches designed for high Knudsen number flows. Kinetic approaches solving the Boltzmann equation are numerically expensive and are limited to very simple problems at current stage. The widely used kinetic approach is the direct simulation Monte Carlo (DSMC) method that avoids solving the Boltzmann equation mathematically by tracking the microscopic motions of particles [3]. The DSMC method is efficient for high-speed high Knudsen number flows, and becomes inefficient for low-speed and near continuum flows because of the statistical noise problem and the cell size restriction. Unfortunately, micro-scale gas flows are usually low speed flows. To resolve the statistical noise problem for the low speed flows, several authors have proposed various modifications to the standard DSMC method. These include the information preservation method [4], the low variance reduction approach [5], and the low Mach number DSMC algorithm [6].

The information preservation (IP) method preserves the information at the macroscopic level in particles simulated in the DSMC method. The preserved information is transferred, 
updated, and sampled to obtain the mean flow information. Thus the statistical noise caused by the thermal velocity is well controlled. The low variance reduction method (LVDSMC) is aimed at the molecular velocity distribution function that is expressed as a sum of an equilibrium Maxwell contribution and the deviation contribution. The LVDSMC method simulates the deviation from equilibrium and thus the numerical efficiency is improved for low-speed flows. The low Mach number DSMC (LMDSMC) method assigns the equilibrium velocity distribution in simulated particles and employs particle weights to represent the deviation from equilibrium. These modified DSMC approaches have achieved success for low-speed flows and are expected to work for general flows by further developments.

In fact, the statistical noise is one challenge for DSMC simulations. Another challenge is the cell size restriction for near continuum flows. The DSMC method requires that the cell size should be less than one molecular mean free path. Hence, low Knudsen number flows are also numerically expensive for DSMC simulations. In order to employ large cells in DSMC, Bird has proposed the subcell technique [3] and the nearest particle selection technique [7] by limiting the separation distance between collision pairs. Another strategy is to enlarge the effective cell size by including flow field information during particle collisions [8].

In this study, we propose a cell size relaxed information preservation method for micro-scale gas flows where the statistical noise and cell size restriction are both considered. The details of the method are described in Section 2. The method is evaluated and applied to study the 2D lid-driven cavity flows in Section 3. Finally, we summarize the main conclusions in Section 4.

\section{AN EFFICIENT PARTICLE APPROACH}

The direct simulation Monte Carlo method is a widely used approach for simulating transitional flows. It simulates particle's microscopic motions and is valid for gas flows in the entire flow regimes in principle. However, it is numerically expensive for low-speed small Knudsen flow simulations. For low-speed flows, the information preservation method is very effective to control the statistical noise. For small Knudsen number flows, the cell size relaxed scheme is able to extend the cell size to 10 molecular mean free paths. Hence these two techniques are combined to develop an efficient approach for micro-scale gas flow simulations. We start the description of the method from the standard DSMC method.

\subsection{Direct Simulation Monte Carlo Method}

The direct simulation Monte Carlo (DSMC) method is a particle simulation approach proposed by Bird [3]. The basic idea of the method is to simulate the microscopic motions of a large number of particles and to obtain the flow field information by statistically averaging the microscopic motions.
It does not solve directly the Boltzmann equation, but has been approved that the method is equivalent to solving the Boltzmann equation.

The DSMC method is based on three important assumptions. First, the binary collision assumption allows the multi-body collisions to be neglected, which is consistent with the Boltzmann equation. Second, the chaos assumption allows the particles to be randomly selected during particle collisions. Third, the interactions among molecules are assumed instantaneous. This last assumption is the basis for the key approximation in the DSMC method, the decoupling between particle collisions and motions. The decoupling is very important for the numerical efficiency because the exact trajectory of a particle is not needed and the collisions can be treated statistically.

Because the decoupling between collisions and motions is an approximation, the DSMC method requires that the cell size should be less than one molecular mean free path and the time step be smaller than one collision time. Therefore a typical DSMC simulation consists of two key steps: particle collisions and particle motion. There are other treatments, of course, to make a DSMC simulation complete. One is that each simulated particle should carry its microscopic information such as position, velocity and internal energy. Another is that the particle information is sampled and averaged to obtain the macroscopic flow information. Because the sample size is limited, a DSMC simulation always has statistical noise.

The cost for a DSMC simulation mainly depends on three factors: the number of simulated cells, the simulation time steps, and the sampling size in a cell. For low-speed flows, a large sampling size is required because the statistical noise is independent of the flow speed and the noise to signal ratio is large for low-speed flows. For near-continuum flows, a large number of cells and time steps are required, which may exceed the allowed memory in some machines. Therefore, for microscale gas flows, the challenges for DSMC simulations are the statistical noise, the cell size, and the time step.

\subsection{Information Preservation Method}

The information preservation (IP) method is a particle approach based on the DSMC method with an intention to resolve the statistical noise problem. The IP method preserves additional information in the particles simulated in the DSMC method. The preserved information is case dependent, but is in the macroscopic level. For instance, in the original version of the IP method [4], each particle owns microscopic velocity and the "information velocity". The microscopic velocity is used to track the particle's microscopic motions and the "information velocity" is employed to sample the flow information. Since the "information velocity" does not have thermal velocity part, the sampled flow information has very small statistical noise.

The IP method has been developed to include "information temperature" in particles and "information velocity", "information temperature", and "information density" in cells 
to account for general gas flows. General equations have also been derived to update the preserved information using the Maxwell's equation of change:

$$
\frac{\partial}{\partial t}(n \bar{Q})+\nabla \cdot(n \overline{\mathbf{c} Q})=\Delta[Q]
$$

where $Q$ is the quantity related to a particle, and $\Delta[Q]$ denotes the change of $Q$ during particle collisions. The preserved information follows these equations:

$$
\begin{gathered}
\frac{\partial}{\partial t}(n m)+\nabla \cdot(n m \overline{\mathbf{V}})=0 \\
\frac{\partial}{\partial t}(n m \overline{\mathbf{V}})+\nabla \cdot(n m \overline{\mathbf{c V}})=-\nabla \cdot\left(n m \overline{\mathbf{c}^{\prime} \mathbf{c}^{\prime \prime \prime}}\right) \\
\frac{\partial}{\partial t}\left(\frac{1}{2} n m \overline{\left(V^{2}+3 R T\right)}\right)+\nabla \cdot\left(\frac{1}{2} n m \overline{\mathbf{c}\left(V^{2}+3 R T\right)}\right)=\nabla \cdot\left(\frac{1}{2} n m \overline{\mathbf{c}^{\prime}\left(V^{2}+3 R T-c^{2}\right)}\right)
\end{gathered}
$$

where $\mathrm{V}$ is the "information velocity", $\mathrm{c}$ is the microscopic velocity. If $\mathrm{c}_{0}$ is the mean flow velocity, then $\mathbf{c}^{\prime}=\mathbf{c}-\mathbf{c}_{\mathbf{0}}$ and $\mathbf{c}^{\prime \prime \prime}=\mathbf{c}-\mathbf{V}$.

It is noted that there are average or statistical terms in the above equations and these terms should be modeled [9]. The latest model is the one developed by Masters and Ye [10]. In order to capture the viscosity and heat conductivity of the flow, the preserved information is also updated during particle collisions where conservation of momentum and energy is enforced. The latest collision model is the one proposed by Sun and Boyd [11].

The IP method is very efficient for low-speed flows as compared with the standard DSMC method. The IP method has been applied to study some micro gas flows and further studies are expected to include nonequilibrium among energy components.

\subsection{Cell-Size Relaxed DSMC Method}

The standard DSMC method requires that the cell size should be less than one molecular mean free path, which is very tough for near continuum flow simulations. It has been analyzed that the cell size restriction comes from the pickup treatment of the collision particle pair [12]. The collision pair is randomly selected within the computational cell in the standard DSMC method. Namely, the location difference of the pair is not considered. In fact, the flow field is not uniform within the cell generally. If the difference of the flow field information at particle location is properly involved in the collision, the numerical error caused by the finite cell size could be controlled.

Sun et al. [12] has proposed a cell-size relaxed scheme that allows a cell size as large as 10 mean free paths. The scheme consists of several steps.

1) The cell-based flow information (velocity and temperature) is evaluated by sampling particles' information.

2) The flow information at particle location is estimated using the least-square method.

3) The post-collision velocity is calculated by modifying the VHS collision model:

$$
\begin{aligned}
& \left(\frac{\vec{V}_{1}-\vec{U}_{1}}{\sqrt{2 k T_{1}}}\right)^{\prime}=\frac{1}{2}\left(\frac{\vec{V}_{1}-\vec{U}_{1}}{\sqrt{2 k T_{1}}}+\frac{\vec{V}_{2}-\vec{U}_{2}}{\sqrt{2 k T_{2}}}\right)+\frac{1}{2}\left|\frac{\vec{V}_{1}-\vec{U}_{1}}{\sqrt{2 k T_{1}}}-\frac{\vec{V}_{2}-\vec{U}_{2}}{\sqrt{2 k T_{2}}}\right| \cdot \vec{e}(\theta, \phi) \\
& \left(\frac{\vec{V}_{2}-\vec{U}_{2}}{\sqrt{2 k T_{2}}}\right)^{\prime}=\frac{1}{2}\left(\frac{\vec{V}_{1}-\vec{U}_{1}}{\sqrt{2 k T_{1}}}+\frac{\vec{V}_{2}-\vec{U}_{2}}{\sqrt{2 k T_{2}}}\right)-\frac{1}{2}\left|\frac{\vec{V}_{1}-\vec{U}_{1}}{\sqrt{2 k T_{1}}}-\frac{\vec{V}_{2}-\vec{U}_{2}}{\sqrt{2 k T_{2}}}\right| \cdot \vec{e}(\theta, \phi)
\end{aligned}
$$

where $\mathrm{U}$ and $\mathrm{T}$ is the flow velocity and temperature at particle's location, respectively.

4) The velocity of all particles in the cell is adjusted to ensure the conservation of momentum and energy.

$$
\vec{V}_{i}^{\prime \prime}=\frac{\vec{V}_{i}^{\prime}-\vec{\lambda}}{\tau}
$$

where

$$
\begin{array}{r}
\vec{\lambda}=\frac{1}{n}\left(\sum_{i=1}^{n} \vec{V}_{i}^{\prime}-\tau \cdot \sum_{i=1}^{n} \vec{V}_{i}\right) \\
\tau^{2}=\frac{n \cdot \sum_{i=1}^{n} \vec{V}_{i}^{\prime 2}-\left(\sum_{i=1}^{n} \vec{V}_{i}^{\prime}\right)^{2}}{n \cdot \sum_{i=1}^{n} \vec{V}_{i}^{2}-\left(\sum_{i=1}^{n} \vec{V}_{i}\right)^{2}}
\end{array}
$$

It is also noted that slight modification of the flow information at particle location could improve the accuracy of the modified DSMC method. The trick is to replace the calculated flow information at particle location with the following information.

$$
\begin{gathered}
U_{1}^{m}=U_{1}+c_{u} \cdot\left(U_{1}-U_{2}\right) \\
U_{2}^{m}=U_{2}-c_{u} \cdot\left(U_{1}-U_{2}\right) \\
T_{1}^{m}=T_{1} \cdot\left(\frac{T_{1}}{T_{2}}\right)^{c_{T}} \\
(22 \\
T_{2}^{m}=T_{2} \cdot\left(\frac{T_{2}}{T_{1}}\right)^{c_{T}}
\end{gathered}
$$

where subscripts 1 and 2 refer to the particle pair and $c_{u}, c_{T}$ are constants having small value.

Several examples have shown that the accuracy of the DSMC method could be greatly improved so that a simulation having a cell size of 10 mean free paths could give pretty satisfied results.

\subsection{Cell-Size Relaxed IP Method}

In the cell-size relaxed DSMC method, the cell-based information is evaluated by sampling the particles' microscopic information that could exhibit large statistical noise. Since the IP method has the cell-based information available during the simulation, it is beneficial to implement the cell-size relaxed technique in the IP method.

In the cell-size relaxed IP method, the flow information is then evaluated based on the preserved information. The post collision values for the preserved information are calculated as follows:

$$
U_{1}^{\prime}=\frac{1+C_{\mu} \cdot \cos \theta}{2}\left(U_{1}-U_{c 1}\right)+\frac{1-C_{\mu} \cdot \cos \theta}{2}\left(U_{2}-U_{c 2}\right)+U_{c 1}
$$




$$
\begin{aligned}
U_{2}^{\prime}= & \frac{1+C_{\mu} \cdot \cos \theta}{2}\left(U_{2}-U_{c 2}\right)+\frac{1-C_{\mu} \cdot \cos \theta}{2}\left(U_{1}-U_{c 1}\right)+U_{c 2} \\
T_{1}^{\prime}= & \frac{1+C_{k} \cdot \cos \theta}{2}\left(T_{1}-T_{c 1}\right)+\frac{1-C_{k} \cdot \cos \theta}{2}\left(T_{2}-T_{c 2}\right)+T_{c 1} \\
& +\frac{\left(V_{1}-V_{2}\right)^{2}-\left(C_{\mu} \cdot \cos \theta\left(V_{1}-V_{2}\right)+\left(1-C_{\mu} \cdot \cos \theta\right)\left(V_{c 1}-V_{c 2}\right)\right)^{2}}{4 \xi R} \\
T_{2}^{\prime}= & \frac{1+C_{k} \cdot \cos \theta}{2}\left(T_{2}-T_{c 2}\right)+\frac{1-C_{k} \cdot \cos \theta}{2}\left(T_{1}-T_{c 1}\right)+T_{c 2} \\
& +\frac{\left(V_{1}-V_{2}\right)^{2}-\left(C_{\mu} \cdot \cos \theta\left(V_{1}-V_{2}\right)+\left(1-C_{\mu} \cdot \cos \theta\right)\left(V_{c 1}-V_{c 2}\right)\right)^{2}}{4 \xi R}
\end{aligned}
$$

where $\theta$ is the deflection angle and $\xi$ is the number of degrees of freedom.

It is expected that cell-size relaxed IP method will exhibit much smaller statistical noise as compared with the DSMC and the cell size restriction could be relaxed.

\section{2D LID-DRIVEN CAVITY FLOW AT LOW REYNOLDS NUMBER}

The cell-sized relaxed IP scheme is evaluated using the 2D lid-driven cavity flow that is a classical problem in the fluid mechanics [13,14].

The first case is a low Mach small Knudsen number flow where $\mathrm{Ma}=0.005$ and $\mathrm{Kn}=0.006$. At this small $\mathrm{Kn}$ number, the CFD solution solving the Navier-Stokes equation is used to compare with the IP simulation results. Figure 1 shows the comparison between the particle and CFD results for the field information and Fig. 2 shows the surface pressure comparison. Clearly, all the results including the streamlines, velocity and pressure contours agree very well for this case. It is almost impossible for the standard DSMC method to solve this problem because of the computational cost.

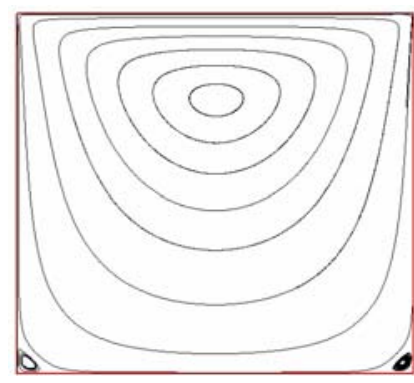

(a) streamlines, IP

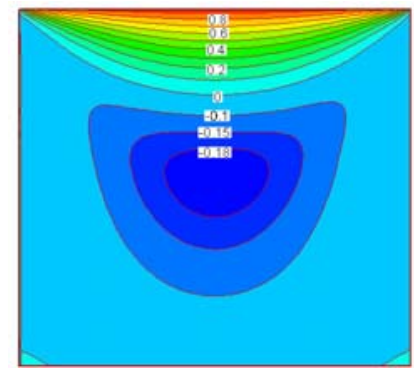

(c) U velocity, IP

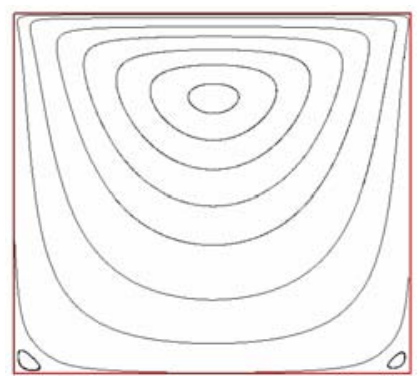

(b) streamlines, CFD

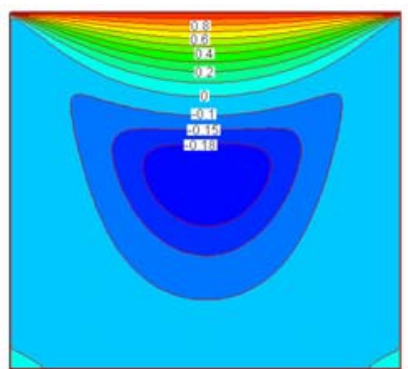

(d) U velocity, CFD

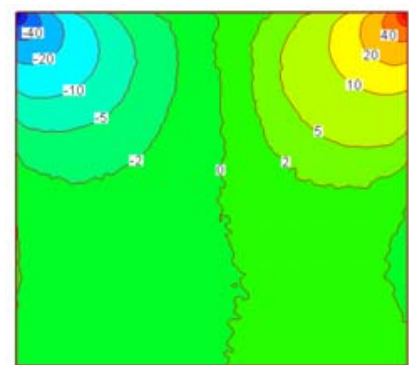

(e) pressure, IP

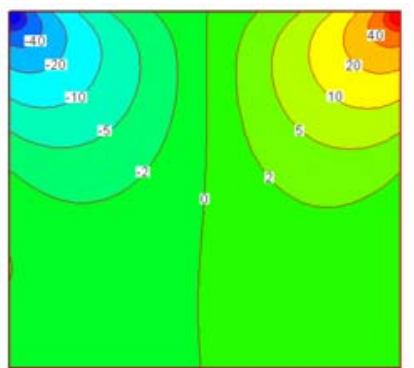

(f) pressure, CFD
FIGURE 1. Comparison between IP results and CFD solution for the driven cavity flow when $\mathrm{Ma}=0.005$ and $\mathrm{Kn}=0.006$.

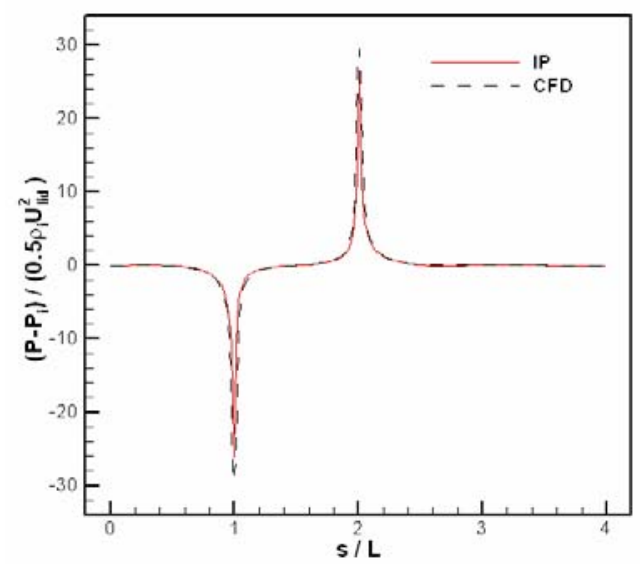

FIGURE 2. Comparison of surface pressure between IP results and CFD solution for the driven cavity flow when $\mathrm{Ma}=0.005$ and $\mathrm{Kn}=0.006$.

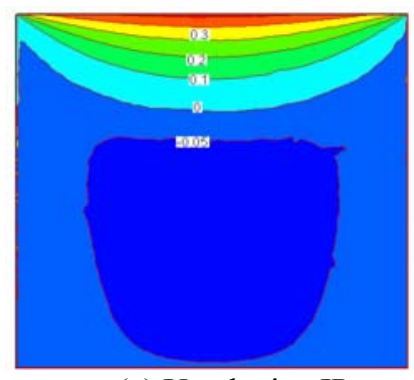

(a) U velocity, IP

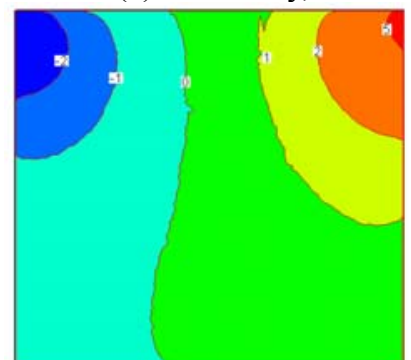

(c) pressure, IP

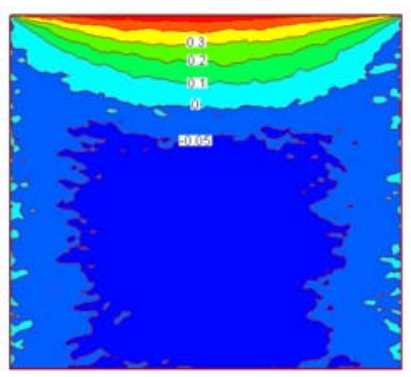

(b) U velocity, DSMC

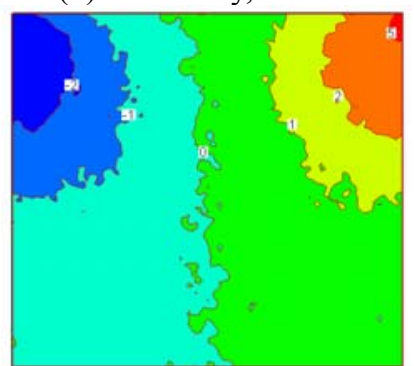

(b) pressure, DSMC
FIGURE 3. Comparison between IP and DSMC results for the driven cavity flow when $\mathrm{Ma}=0.2$ and $\mathrm{Kn}=0.25$. 
The second case has a large Ma and Kn numbers where $\mathrm{Ma}=0.2$ and $\mathrm{Kn}=0.25$. At this setup, the DSMC results can be obtained with not too many sampling size. The agreement between the IP and DSMC results is very well although the DSMC shows severe statistical noise as shown in Figure 3 and Figure 4.

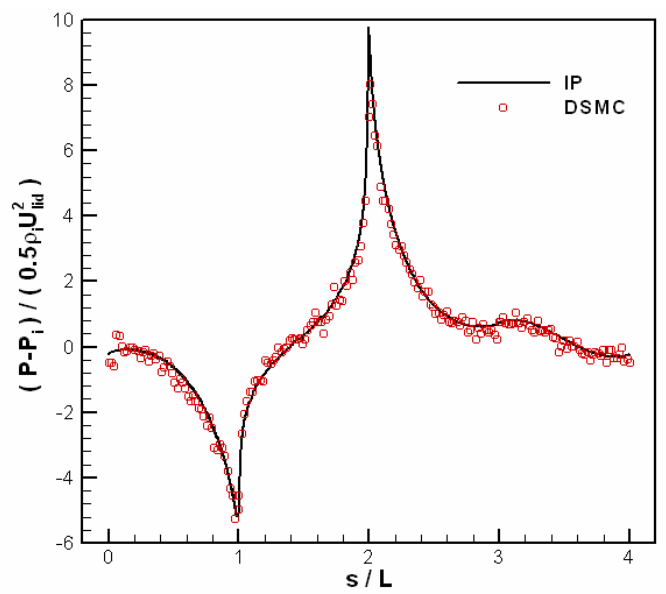

FIGURE 4. Comparison of surface pressure between IP and $\mathrm{DSMC}$ results for the driven cavity flow when $\mathrm{Ma}=0.2$ and $\mathrm{Kn}=0.25$.

As the cell-size relaxed IP method has been tested with the CFD and DSMC methods, the method is used to predict the flows in the driven cavity at various Kn numbers when the Reynolds number is fixed at 1.0. Figure 5 shows the streamlines in the cavity. It shows that there are small corner vortices at low Knudsen numbers and the vortices shrink and even disappear when the Knudsen number increases. Figure 6 shows the U-velocity contours. The velocity is normalized using the lid velocity. It shows that the velocity variation decreases when the Knudsen number increases. Similar trend is observed for the pressure as shown in Fig. 7 where the pressure is nondimensionalized using the dynamic pressure. The surface pressure is shown in Fig. 8. It shows that the pressure decreases sharply when the Knudsen number increases, but the variation regions are enlarged since the mean free path is increased with the Kn number. The rarefied gas effects can be well represented by the lid slip velocity as shown in Fig. 9. It shows that the slip velocity increases with the $\mathrm{Kn}$ number, but the slip becomes very large near the corners even when the Knudsen number is less than 0.01 . This indicates that the corners or the singular points play very critical role in the flow phenomena.

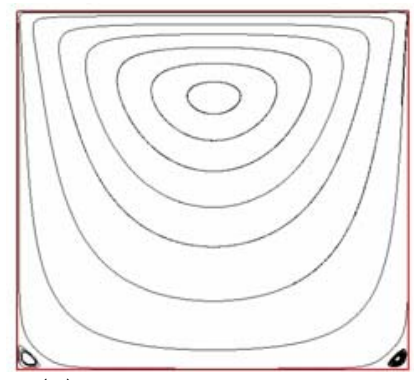

(a) $\mathrm{Ma}=0.005, \mathrm{Kn}=0.00625$

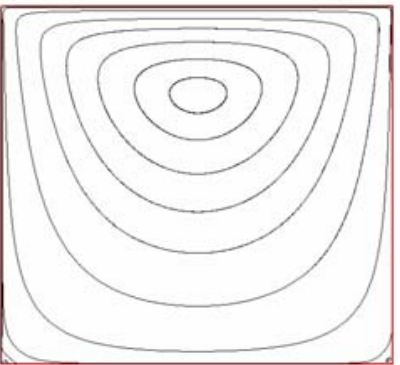

(c) $\mathrm{Ma}=0.02, \mathrm{Kn}=0.025$

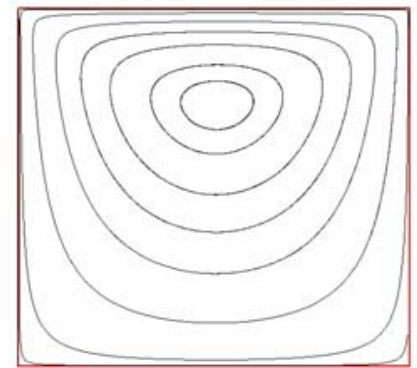

(e) $\mathrm{Ma}=0.1, \mathrm{Kn}=0.125$

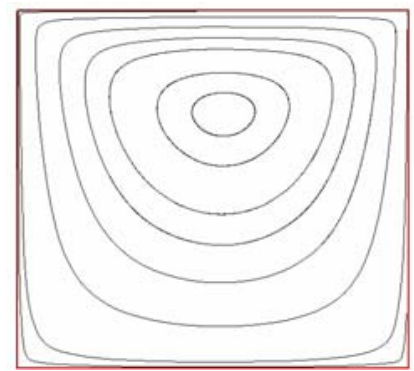

(g) $\mathrm{Ma}=0.4, \mathrm{Kn}=0.5$

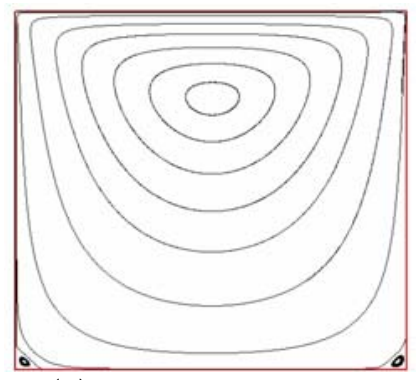

(b) $\mathrm{Ma}=0.01, \mathrm{Kn}=0.0125$

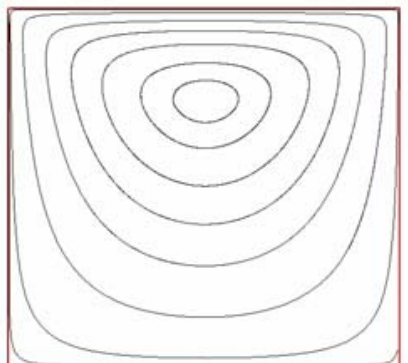

(d) $\mathrm{Ma}=0.05, \mathrm{Kn}=0.0625$

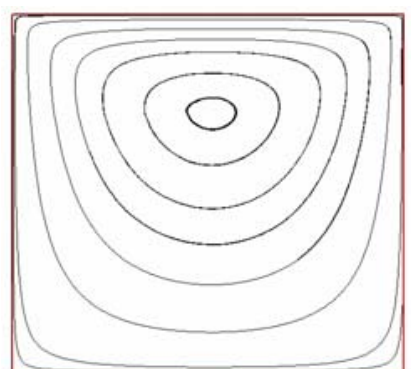

(f) $\mathrm{Ma}=0.2, \mathrm{Kn}=0.25$

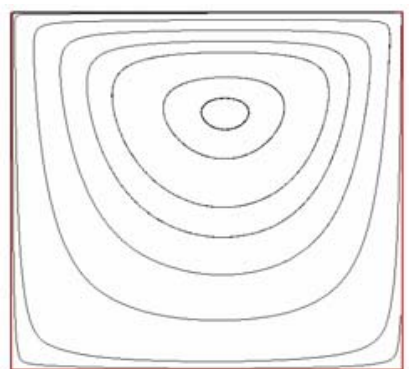

(h) $\mathrm{Ma}=0.8, \mathrm{Kn}=1$
FIGURE 5. Streamlines in the driven cavity when $\mathrm{Re}=1$ 


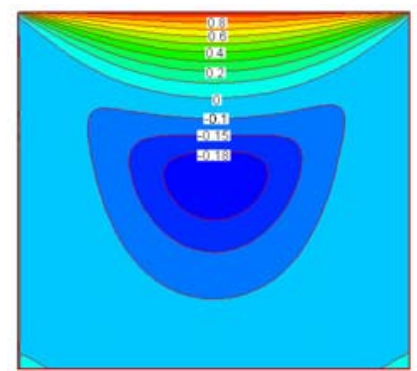

(a) $\mathrm{Ma}=0.005, \mathrm{Kn}=0.00625$

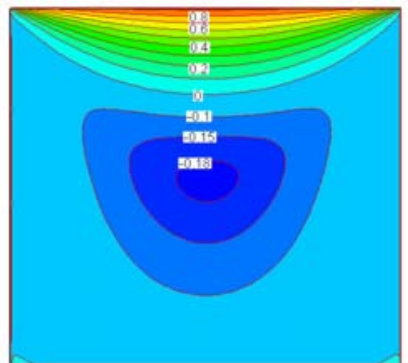

(b) $\mathrm{Ma}=0.01, \mathrm{Kn}=0.0125$

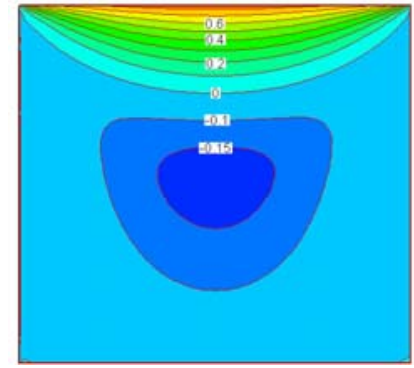

(c) $\mathrm{Ma}=0.02, \mathrm{Kn}=0.025$

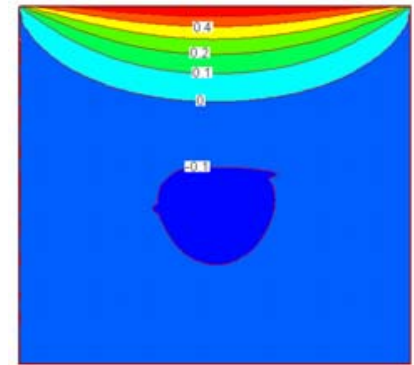

(e) $\mathrm{Ma}=0.1, \mathrm{Kn}=0.125$

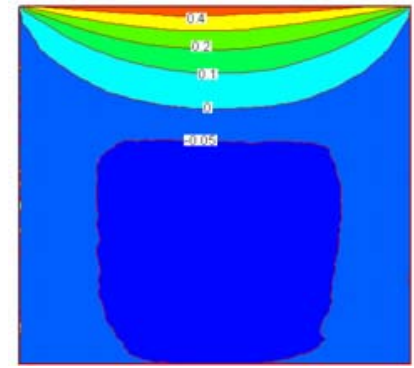

(g) $\mathrm{Ma}=0.4, \mathrm{Kn}=0.5$

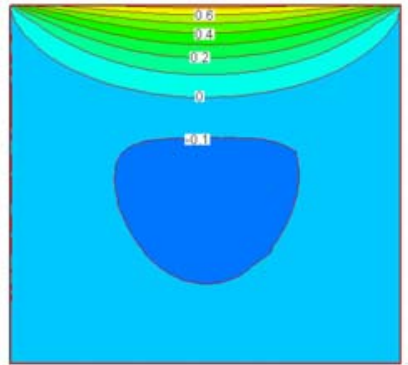

(d) $\mathrm{Ma}=0.05, \mathrm{Kn}=0.0625$

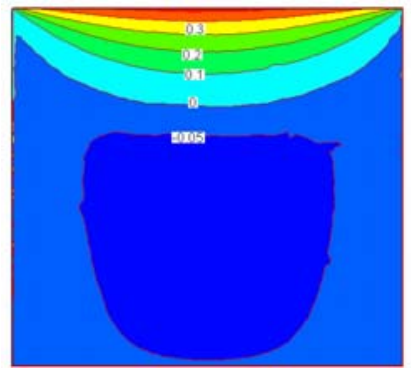

(f) $\mathrm{Ma}=0.2, \mathrm{Kn}=0.25$

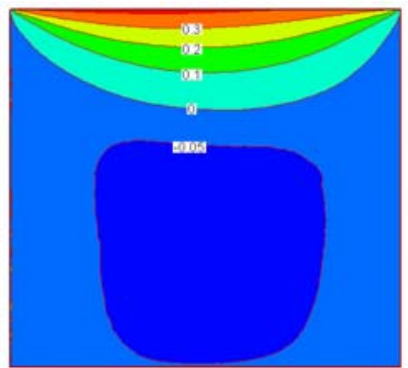

(h) $\mathrm{Ma}=0.8, \mathrm{Kn}=1$

FIGURE 6. U-velocity contours in the driven cavity when $\mathrm{Re}=1$

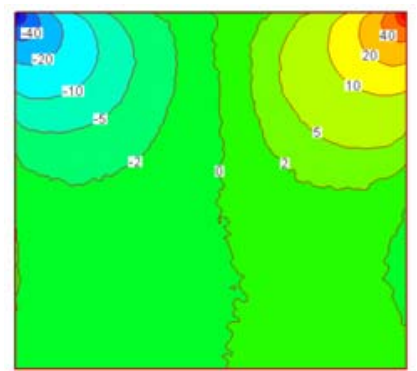

(a) $\mathrm{Ma}=0.005, \mathrm{Kn}=0.00625$

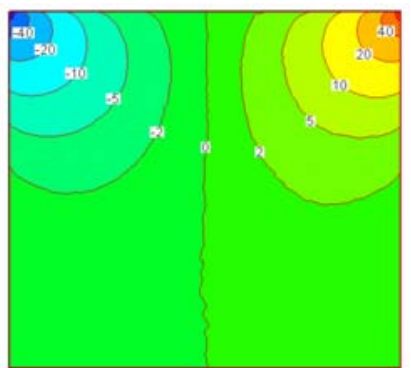

(b) $\mathrm{Ma}=0.01, \mathrm{Kn}=0.0125$

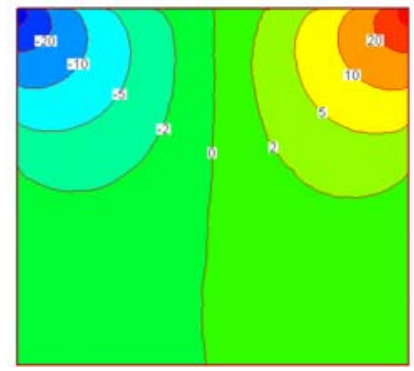

(c) $\mathrm{Ma}=0.02, \mathrm{Kn}=0.025$
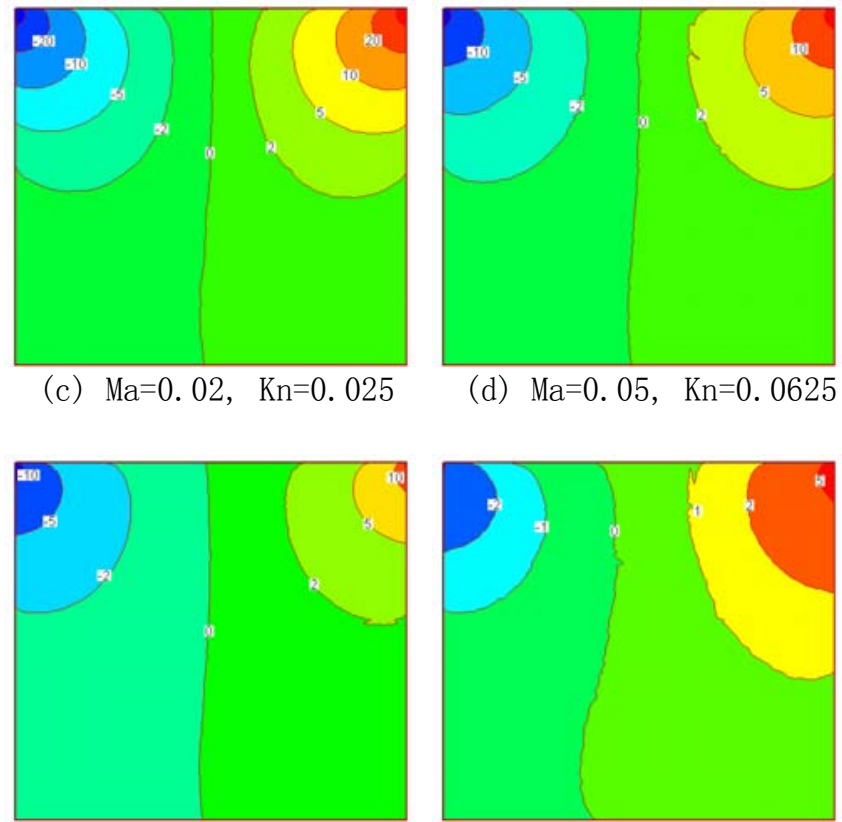

(e) $\mathrm{Ma}=0.1, \mathrm{Kn}=0.125$

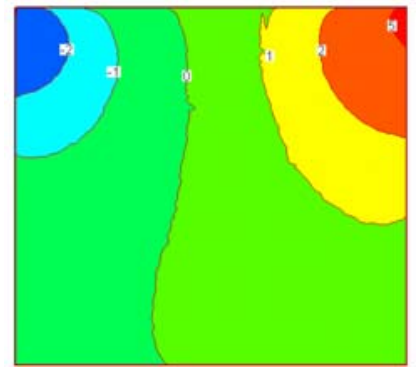

(f) $\mathrm{Ma}=0.2, \mathrm{Kn}=0.25$

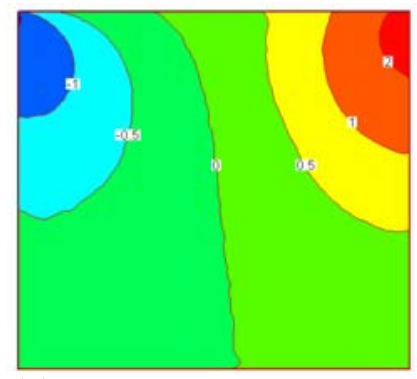

(g) $\mathrm{Ma}=0.4, \mathrm{Kn}=0.5$

(h) $\mathrm{Ma}=0.8, \mathrm{Kn}=1$

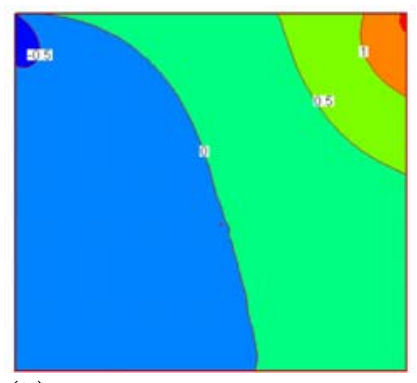

FIGURE 7. Pressure contours in the driven cavity when $\mathrm{Re}=1$ 


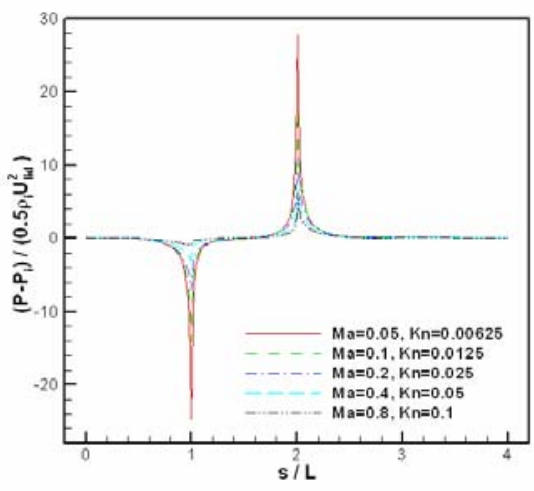

(a) pressure coefficient

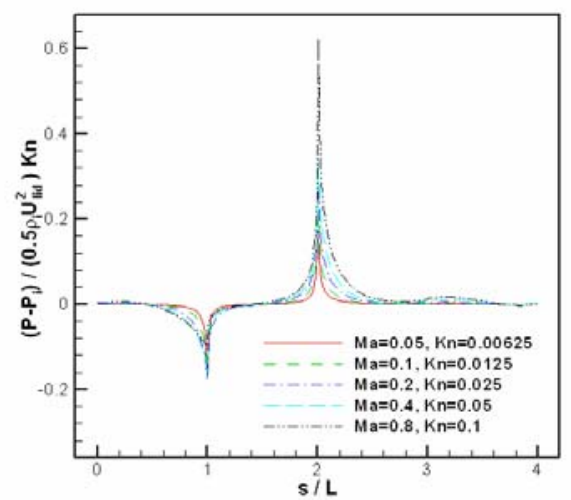

(b) pressure coefficient multiplied by the Kn number

FIGURE 8. Surface pressure in the driven cavity when $\mathrm{Re}=1$

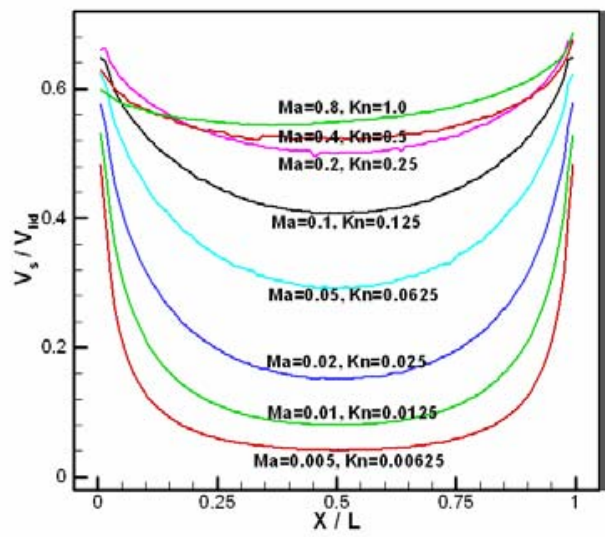

FIGURE 9. Slip velocity on the driven lid when $\mathrm{Re}=1$

\section{CONCLUSIONS}

The micro-scale gas flows are usually at low-speed but exhibit rarefied gas effects, which is difficult for numerical simulations. We have developed a cell-sized relaxed IP method for this purpose. The new method can use a cell size that is larger than the molecular mean free path and exhibits much smaller statistical noise as compared with the DSMC method.
The 2D lid-driven cavity flows show that the developed method is able to simulate low-speed small Knudsen number flows. In addition, our simulations found that the singular points caused the flow to slip even at very small Knudsen number.

\section{ACKNOWLEDGMENTS}

The authors would like to acknowledge the support from National Natural Science Foundation of China (Grants No. 90816012, No. 10621202, and No. 10742001).

\section{REFERENCES}

[1] G. E. Karniadakis, A. Beskok, N. Aluru, Microflows and Nanoflows: Fundamentals and Simulation, Springer, New York, 2005

[2] C. Cai, I. D. Boyd, J. Fan, and F. V. Candler, "Direct simulation methods for low-speed microchannel flows", J. Thermophys. Heat Transfer 14(3)(2000), 368-378

[3] G. A. Bird, Molecular gas dynamics and the direct simulation of gas flows, Clarendon, Oxford, 1994

[4] J. Fan and C. Shen. "Statistical simulation of low-speed rarefied gas flows", J. Comput. Phys., 167(2001), 393-412

[5] L. Baker and N. Hadjiconstantinou, "Variance reduction for Monte Carlo solutions of the Boltzmann equation", Phys. Fluids, 17 (2005), 051703

[6] J. Chun and D. Koch, "A direct simulation Monte Carlo method for rarefied gas flows in the limit of small Mach number", Phys. Fluids, 17 (2005), 107107

[7] G. A. Bird, "Sophisticated versus simple DSMC", in Rarefied Gas Dynamics (Ed. by M. S. Ivanov and A. K. Rebrov), 2007, 815-820

[8] Q. Sun, H. Liu, J. Fan, "Development of a cell sized relaxed scheme for the direct simulation Monte Carlo method", in Rarefied Gas Dynamics (Ed. by T. Abe), 2009, 311-316

[9] Q. Sun and I D Boyd, "Theoretical development of the information preservation method for strongly nonequilibrium gas flows", AIAA 2005-4828

[10] N. D. Masters and W. Ye, "Octant flux splitting information preservation DSMC method for thermally driven flows", J. of Comput. Physics, 2007(226), 2044-2062

[11] Q. Sun and I. D. Boyd, "A direct simulation method for subsonic, micro-scale gas flow", J. of Comput. Physics, 2002(179), 400-425

[12] Q. Sun, H. Liu, J. Fan, "Development of a cell size relaxed scheme for the direct simulation Monte Carlo method", in Rarefied Gas Dynamics, 1084(2009), 311-316

[13] S. Mizzi, D. R. Emerson, S. K. Stefanov, R. W. Barber and J. M. Reese, "Effects of Rarefaction on Cavity Flow in the Slip Regime", J. of Comput. \& Theoretical Nanoscience, 2007(4), $817-822$

[14] J. Jiang, J. Fan and C. Shen, "Statistical simulation of micro-cavity flows", in Rarefied Gas Dynamics: 23rd International Symposium, edited by A. D. Ketsdever and E. P. Muntz, 2003, 784 